Article

\title{
Polyelectrolytes: Influence on Evaporative Self-Assembly of Particles and Assembly of Multilayers with Polymers, Nanoparticles and Carbon Nanotubes
}

\section{Irina Marchenko ${ }^{1}$, Alexey Yashchenok ${ }^{2,3}$, Sergey German ${ }^{2}$, Olga Inozemtseva ${ }^{2}$,} Dmitry Gorin ${ }^{2,3}$, Tatiana Bukreeva ${ }^{1}$, Helmuth Mohwald ${ }^{3}$ and Andre Skirtach ${ }^{3, *}$

1 Institute of Crystallography, Russian Academy of Sciences, Moscow 119333, Russia; E-Mails: irina.marchenko@mpikg.mpg.de (I.M.); tanika71@mail.ru (T.B.)

2 Saratov State University, Saratov, 410012, Russia; E-Mails: Alexey.Yashchenok@mpikg.mpg.de (A.Y.); s.german@mail.ru (S.G.); Olga.Inozemtseva@mpikg.mpg.de (O.I.); Dmitry.Gorin@mpikg.mpg.de (D.G.)

3 Max-Planck Institute of Colloids and Interfaces, Golm, Potsdam, D-14476, Germany; E-Mail: moehwald@mpikg.mpg.de (H.M.)

* Author to whom correspondence should be addressed; E-Mail: skirtach@mpikg.mpg.de; Tel.: +49-331-567-9409; Fax: +49-331-567-9202.

Received: 14 October 2010; in revised form: 19 November 2010 / Accepted: 6 December 2010 / Published: 9 December 2010

\begin{abstract}
Assembling polyelectrolyte multilayers in a bottom-up approach is reported for polymers, particles, nanoparticles, and carbon nanotubes. Effects of polyelectrolyte multilayers on evaporative self-assembly of particles, which are of interest to a number of applications including photonic crystals, films and substrates, are investigated. Polyelectrolyte multilayer coatings bring multifunctionality to spherical particles and planar films. Studying the construction of polyelectrolyte assemblies is convenient in the planar layout: it is reported here for incorporation of gold and magnetic nanoparticles as well as of carbon nanotubes. Gold nanoparticles concentration is controlled within the films. Potential applications of both spherical structures and planar films are highlighted.
\end{abstract}

Keywords: self-assembly; polyelectrolytes; silica particles; carbon nanotubes; gold and magnetic nanoparticles 


\section{Introduction}

Nanotechnology approaches offer novel avenues for building films, capsules and drug delivery carriers [1]. The layer-by-layer (LbL) assembly of polyelectrolyte multilayers (PEM) [2-6], which applies nanotechnology tools, is an attractive method for fabrication of various structures. Based on the electrostatic interaction, the $\mathrm{LbL}$ absorption is a simple but powerful method for functionalization of colloidal particles [7,8] and planar films [9-13], which lead to a variety of interesting applications ranging from implant coatings [14] to growth factor incorporation [15] and bone and dental applications [16,17]. Its particular advantage stems from possibilities of incorporation of various materials, for example, polymers, nanoparticles, proteins, etc. during the build-up. First and foremost, planar films [18-23], as well as spherical particles and capsules [24-30], benefit from the multifunctionality that incorporation of diverse materials brings.

Functionalization by nanoparticles [31,32] is of interest for diverse applications both on planar films and spherical particles. For example, assemblies of gold nanoparticles incorporated into polymeric films exhibit sensor properties [33,34], they can be used to improve the mechanical stability of polymeric microcapsules [35,36], and for remote activation of microcapsules [37-42]. Demonstration of remote release inside living cells [43] enabled tracing intracellular processes relevant to immunology [44]. Besides, aggregates of nanoparticles embedded within biocompatible films can be activated by a near-IR laser [21-23]; these effects are based on nanoplasmonic heating of nanoparticles [45-57]. Additionally, magnetic nanoparticles enable positioning of polyelectrolyte capsules [58], while on the other hand activation is also possible [59]. Carbon nanotubes [60-65] are attractive candidates for functionalization, too, because, on the one hand, they enhance mechanical properties [66], while on the other hand, they are found to be extremely efficient absorption centers [67]. All these materials are attractive candidates for functionalization of polyelectrolyte multilayers, planar films and spherical microcapsules.

Self-assembly is an interesting method for fabrication of nanostructures [65]. In the last two decades, fabrication of 1D, 2D and 3D architectures [69,70] received significant attention from researchers. These blocks are attractive as novel materials with potential application as components of optoelectronic devices [71], including molecules detectors and photonic crystals [72]. Various methods have been employed for building assemblies based on micro- and nanoparticles such as a spin coating [73], electro deposition [74] and vertical deposition [75,76]. An interesting method for deposition is that based on self-assembly, particularly by controlled evaporation [77]. Two-dimensional arrays of polystyrene particles $(600 \mathrm{~nm})$ coated with polyelectrolytes were presented [78], while ordered arrays of microcapsules in solution can be harnessed to propagate chemical signals in directed and controllable ways, allowing signals to be transmitted over macroscopic distances [79]. Therefore, it is desirable to investigate the influence of polyelectrolyte multilayer coatings on 3D self-assembly of colloidal particles because such materials are of interest for photonic crystal ordering, free-standing structures, membranes and sensors and self-assembled structures in general.

Here we study self-assembly and evaporation driven ordering of silica colloidal particles and investigate the influence of polyelectrolytes on this process. Since polyelectrolyte multilayer polymeric films represent complementary (to spherical particles) and convenient means to study deposition of polymers, we use planar films for investigation of adsorption of polyelectrolytes with a 
variety of nanoparticles and carbon nanotubes. Processes and structures studied in this work can be further applied in combination with cross-linked polymers [80-82], hydrogel construction [83] as well as immobilization of multicompartment microcapsules [84] and microparticles [85] containing enzymes.

\section{Experimental Section}

\subsection{Materials}

Silica particles $\left(\mathrm{SiO}_{2}\right)$ with two sizes $4.80 \pm 0.19 \mu \mathrm{m}$ (large) and $0.585 \pm 0.02 \mu \mathrm{m}$ (small) were purchased from Microparticles $\mathrm{GmbH}$, Germany. Poly(diallyldimethylammonium, chloride) (PDADMAC, 250-300 kDa), poly(styrenesulfonate, sodium salt) (PSS, $70 \mathrm{kDa}$ ), poly(allylamine, hydrochloride) (PAH, $70 \mathrm{kDa}$ ), poly(ethyleneimine) (PEI, 600-1,000 kDa), with tetramethylrhodamine isothiocyanate (TRITC) were purchased from Sigma-Aldrich, Germany. PAH was labeled with TRITC according to the previously reported procedure [86]; briefly, PAH was mixed with TRITC (in $0.1 \mathrm{M}$ carbonate buffer, $\mathrm{pH} 9.0$ with concentration of dye/polymer $=5: 1$ ) followed by dialysis against $0.01 \mathrm{M}$ TRIS-HCl buffer, pH 7.5 (cut-off MW 14,000). Two types of gold nanoparticles were used: citrate stabilized negatively charged nanoparticles (NNPs) with size $20 \mathrm{~nm}$, purchased from Sigma-Aldrich, Germany. 4-(dimethylamino) pyridine (DMAP)) stabilized positively charged nanoparticles (PNPs) were prepared according to previously published methods [87]; they were positively charged and had a mean diameter of 8-10 nm. Water soluble iron oxide $\left(\mathrm{Fe}_{3} \mathrm{O}_{4}\right)$ nanoparticles stabilized by citric and hydrochloric acids were synthesized using the co-precipitation technique [88]. Multiwall carbon nanotubes (MWCNTs) with diameter 110-170 $\mathrm{nm}$ and length 5-9 $\mu \mathrm{m}$ were purchased from Sigma-Aldrich, Germany.

\subsection{Shell Fabrication of Silica Particles}

Absorption of polyelectrolytes on the shells of silica particles was done by the LbL approach. Before deposition of polyelectrolytes onto silica cores, three washing cycles were performed. Then particles were re-dispersed in $0.5 \mathrm{M} \mathrm{NaCl}$ solution of PDADMAC with a concentration of $2 \mathrm{mg} / \mathrm{mL}$ and left in a shaker for 15 minutes, followed by three centrifugation cycles and washing by deionized water. After that, a solution of PSS $(2 \mathrm{mg} / \mathrm{mL}$ in $0.5 \mathrm{M} \mathrm{NaCl})$ was added to the tube for producing of a second polyelectrolyte layer. Particles were constantly shaken for 15 minutes in the PSS solution; followed by three washing steps. As a result of this procedure one polyelectrolyte bi-layer of PDADMAC/PSS was deposited on the surface of silica particles. Subsequent polyelectrolyte layers were deposited using the same procedure as described above. For visualization of small silica particles, one layer of TRITC-PAH $(1 \mathrm{mg} / \mathrm{mL}$ in $0.5 \mathrm{M} \mathrm{NaCl})$ was added on the PSS layer to form the following structure: (PDADMAC/PSS) 4 /PDADMAC/PSS/TRITC-PAH/PSS.

\subsection{Particle Arrays Construction}

Prior to fabrication of coated particle arrays on glass slides the latter were treated with piranha solution (concentrated sulfuric acid and 30\% hydrogen peroxide, 1:3) followed by washing in deionized water and drying in nitrogen flow. CAUTION: Piranha solution reacts violently with 
organic solvents and is a skin irritant. Extreme caution should be exercised when handling the piranha solution. After such a procedure, glass become hydrophilic by hydroxylating the surface, thus increasing the number of silanol groups on the surface. For building particle arrays on the cleaned glass slides, solutions $(\sim 20 \mu \mathrm{L})$ containing particles $\left(0.7 \%\right.$ weight, $\left.\mathrm{SiO}_{2}\right)$ were deposited onto the slide surface and left for drying. For cases when more than 1 layer was formed, the particles forming the next layer were deposited onto the first layer similarly to the procedure described above; similarly the glass slide with particles was left for drying. In case of adsorption of particles in solution, the cleaned and PEI coated slide was immersed into a solution containing coated large silica particles and was left incubating for 15 minutes. In the next step the slide was slowly removed from the solution. Prior to deposition of particles the cleaned glass slide was coated with PEI ( $2 \mathrm{mg} / \mathrm{mL}$ in water).

\subsection{Gold Nanoparticles in Polyelectrolyte Films}

Cleaned glass slides were used as substrates for fabrication of polyelectrolyte films with embedded gold nanoparticles. The substrates were cleaned similarly to the case of particle array construction (Section 2.3). Subsequently, they were dried in nitrogen flow and were immersed in PEI solution ( $2 \mathrm{mg} / \mathrm{mL}$ in water) for 10 minutes to charge the surface of the substrate; followed by washing with deionized water. Then, the substrate was then similarly immersed in PSS and PDADMAC solutions $(2 \mathrm{mg} / \mathrm{mL}$ in $0.5 \mathrm{M} \mathrm{NaCl})$ for the deposition of multilayered polyelectrolyte structures. Gold nanoparticles were adsorbed onto the polyelectrolyte film due to the electrostatic interaction with oppositely charged polyelectrolyte layer. For that, the films were immersed in gold nanoparticle solution for 25 minutes and washed with water. Three samples of gold nanoparticles were prepared: 1 -Films containing NNPs (concentration of nanoparticles $\sim 10^{11} \mathrm{NP} / \mathrm{cm}^{3}$ ); 2 -Films containing PNPs (concentration of nanoparticles $\sim 10^{14} \mathrm{NP} / \mathrm{cm}^{3}$ ); 3-Films containing higher concentration (hc)-PNPs (concentration of nanoparticles $\sim 10^{15} \mathrm{NP} / \mathrm{cm}^{3}$ ). The latter were obtained after removing the supernatant $(\sim 0.95 \mathrm{~mL})$ from the $1 \mathrm{~mL}$ tube of PNPs which was centrifuged for 1 hour at 10,000 rpm. Using this procedure, films with one and two layers of gold nanoparticles were fabricated. The films with one layer of nanoparticles had the following structures: PEI/PSS/PDADMAC/NNPs and $\mathrm{PEI} / \mathrm{PSS} / \mathrm{PDADMAC/PSS/PNPs}$ for negatively and positively charged nanoparticles, respectively. The films with two layers of nanoparticles had the following structures: PEI/PSS/PDADMAC/NNPs/PDADMAC/PSS/PDADMAC/NNPs and PEI/(PSS/PDADMAC/PSS/PNPs)2. PNPs here stand for both Samples 2 and 3. A film without nanoparticles with structure $\mathrm{PEI} /(\mathrm{PSS} / \mathrm{PDADMAC})_{3} / \mathrm{PSS}$ was made for a control.

\subsection{Magnetic Nanoparticles in Polyelectrolyte Films}

Chemically cleaned p-type (100) silicon wafers were used as substrate. The substrates were cleaned by the RCA cleaning procedure [89] with a mixture of $\mathrm{H}_{2} \mathrm{O} / \mathrm{H}_{2} \mathrm{O}_{2} / \mathrm{NH}_{3}$, ratio $5: 1: 1$ at $70{ }^{\circ} \mathrm{C}$ for 15 minutes and washed with deionized water. As a result, the silicon wafers were covered by a silicon oxide layer. Due to this layer, the wafer surface became negatively charged in water at neutral $\mathrm{pH}$. One precursor layer of PEI was deposited on the substrates, which led to higher stability of polyelectrolyte/iron oxide multilayers deposited later on the same substrate. All polyelectrolytes were used at a concentration of $2 \mathrm{mg} / \mathrm{mL}$; PAH and PSS solutions were prepared in $0.5 \mathrm{M} \mathrm{NaCl}$. Multilayers 
were prepared via LbL self assembly using alternate dipping in a polyelectrolyte solution and an iron oxide suspension. Each deposited layer was washed three times with Milli-Q water before starting the next step of multilayer build-up. The obtained nanocomposite films were dried at ambient conditions after the defined number of oppositely charged layers was deposited. The following compositions were synthesized: $\left.\mathrm{PEI} /\left(\mathrm{Fe}_{3} \mathrm{O}_{4}{ }^{-}\right) / \mathrm{PAH}\right)_{16} / \mathrm{PSS}$ and $\mathrm{PEI} /\left(\mathrm{PSS} / \mathrm{Fe}_{3} \mathrm{O}_{4}{ }^{+}\right)_{16} / \mathrm{PSS} . \mathrm{Fe}_{3} \mathrm{O}_{4}{ }^{-}$are those nanoparticles stabilized by citric acid, while $\mathrm{Fe}_{3} \mathrm{O}_{4}{ }^{+}$are nanoparticles protonated in the presence of hydrochloric acid.

\subsection{Fabrication of Films with Carbon Nanotubes}

MWCNTs ( $1 \mathrm{mg}$ ) were dissolved in $2 \mathrm{mg} / \mathrm{mL}$ PSS solution (total volume $1 \mathrm{~mL}$ ). After stabilization by PSS, the nanotubes were adsorbed on the layer terminated with PDADMAC. They were placed on the film by depositing a drop of the MWCNTs containing solution $(\sim 50 \mu \mathrm{L})$ on the substrate (where the first layer was PEI) followed by washing with deionized water after 15 minutes of incubation. Using this procedure, samples PEI/(MWCNTs/PSS/PDADMAC/PSS/PDADMAC/MWCNTs)N with two $(\mathrm{N}=1)$ and four $(\mathrm{N}=2)$ layers of nanotubes were fabricated.

\subsection{Characterization}

Optical microscopy images were recorded by an inverted Leica TCS SP confocal scanning microscope (Leica, Germany) using a 100×/1.4-0.7-oil immersion objective. An UV-vis (ultraviolet-visible range) spectrometer (Agilent 8453) was used for absorption spectra measurements.

Atomic Force Microscopy AFM measurements were performed in air at room temperature using a Nanoscope III Multimode AFM (Digital Instruments, Maynard, Massachusetts, USA) and NTEGRA Spectra (NT-MDT, Russia) operating in the tapping mode.

Quartz crystal microbalance (QCM) measurements were performed using a multisensory ten channel device connected with a computer. 8.86-MHz AT-cut crystals coated with silver films (QCM active area $=0.256 \mathrm{~cm}^{2}$ ) were employed as a substrate for the LbL deposition; the surface of the resonators was rinsed with deionized water before experiments.

In Raman signal measurements, a continuous laser beam was focused on the sample through a confocal Raman microscope (CRM200, WITec, Ulm, Germany) equipped with a piezo-scanner (P-500, Physik Instrumente, Karlsruhe, Germany). The diode-pumped $785 \mathrm{~nm}$ near infra-red laser excitation (Toptica Photonics AG, Graefelfing, Germany) was used in combination with a $100 \times$ oil immersion (Nikon, $\mathrm{NA}=1.25$ ) microscope objective. A laser power between 15-30 mW was used for all measurements. The spectra were acquired using an air-cooled CCD camera (DU401A-DR-DD, Andor, Belfast, North Ireland) with the grating (300 lines $/ \mathrm{mm}$ ) spectrograph (Acton, Princeton Instruments Inc., Trenton, NJ, USA), the spectral resolution was set at $61 / \mathrm{cm}$. The ScanCtrlSpectroscopyPlus software (version 1.38, Witec) was used in the measurement setup. 


\section{Results and Discussion}

Polyelectrolyte multilayers can be assembled on planar substrates and spherical particles. Figure 1 demonstrates an overview of the bottom-up approach used in assembling elaborate structures. The constituent components and materials of the assembly (the bottom row) are shown together with intermediate steps on deposition on planar and spherical structures. In turn, these spherical and planar structures, combined together, are used for self-assembly of layers.

Figure 1. Schematics of the bottom-up assembly of planar films and spherical particles using polyelectrolyte multilayers, particles, nanoparticles and carbon nanotubes (the bottom row and the middle row). Evaporative self-assembly of particles and polyelectrolyte layers is also shown (the top row).

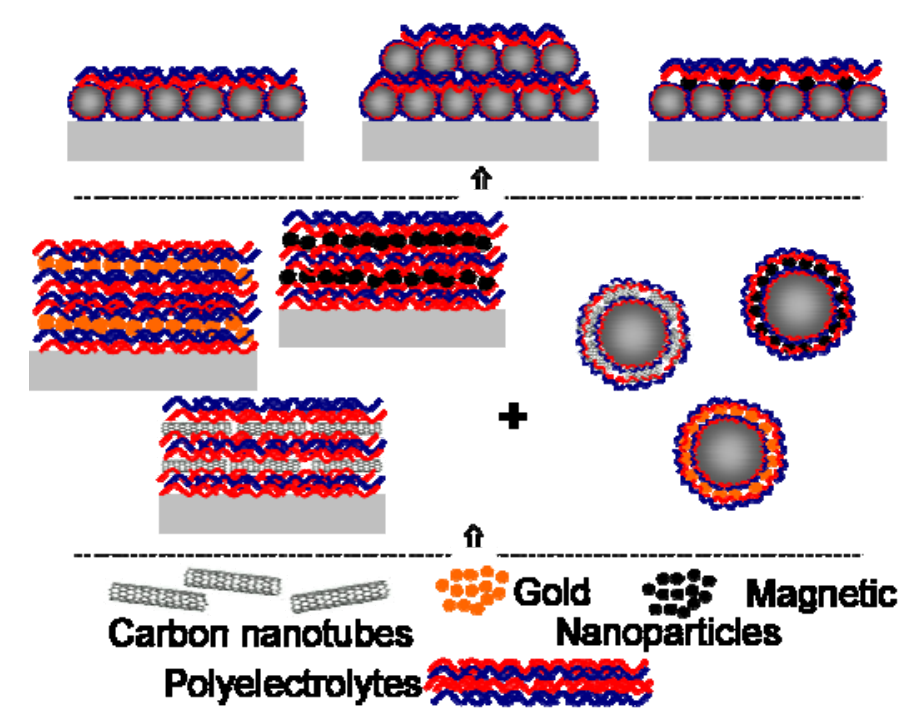

\subsection{Fabrication of Arrays of Silica Colloids and PEM Coated Silica Colloids}

Self-assembly on glass slides upon evaporation of an aqueous solution is studied in our work for microparticle arrays comprised of both polyelectrolyte coated and uncoated silica micro- and nanoparticles. The coatings bring additional functionality to self-assembled particles and may even be able to affect the adsorption. Before self-assembling silica PEM particles, we verified that colloidal silica microparticles without polyelectrolyte layers self-assemble into well-defined arrays by drying the aqueous solution in which the particles were deposited onto the surface (Figure 2(a)). After drying, these silica particles formed a closely packed monolayer (although some spaces between neighboring particles exist, they can be optimized by evaporation and concentration of nanoparticles).

Subsequently, silica particles covered with a PEM layer were self-assembled onto a glass slide. This was done by spreading a defined amount of particle suspension on microscope slides. The transmission CLSM (confocal scanning laser microscope) images of a dried silica monolayer on a microscope slide are presented in Figure 2(b). One can see in this figure that silica PEM particles form a similar pattern as in the case of uncoated silica particles, Figure 2(a). Also, some spaces between particles can be seen, which can be further optimized by drying conditions and concentration of particles. Thus, one can conclude that the presence of polyelectrolytes on the surface does not affect 
self-assembly of particles. In the case when both the substrate and colloidal silica particles are covered with polyelectrolytes, Figure 2(c), a similar self-assembly takes place as in the case when only particles are covered with polyelectrolytes.

Figure 2. Confocal scanning laser microscope (CLSM) transmission microscopy images of evaporative self-assembly of (a) silica and (b) polyelectrolyte coated silica particles on cleaned glass substrates, and (c) polyelectrolyte coated silica particles on a PEI coated glass slide. (d) Adsorption of coated silica particles onto a PEI coated glass slide in an aqueous solution. The scale bars correspond to $20 \mu \mathrm{m}$.
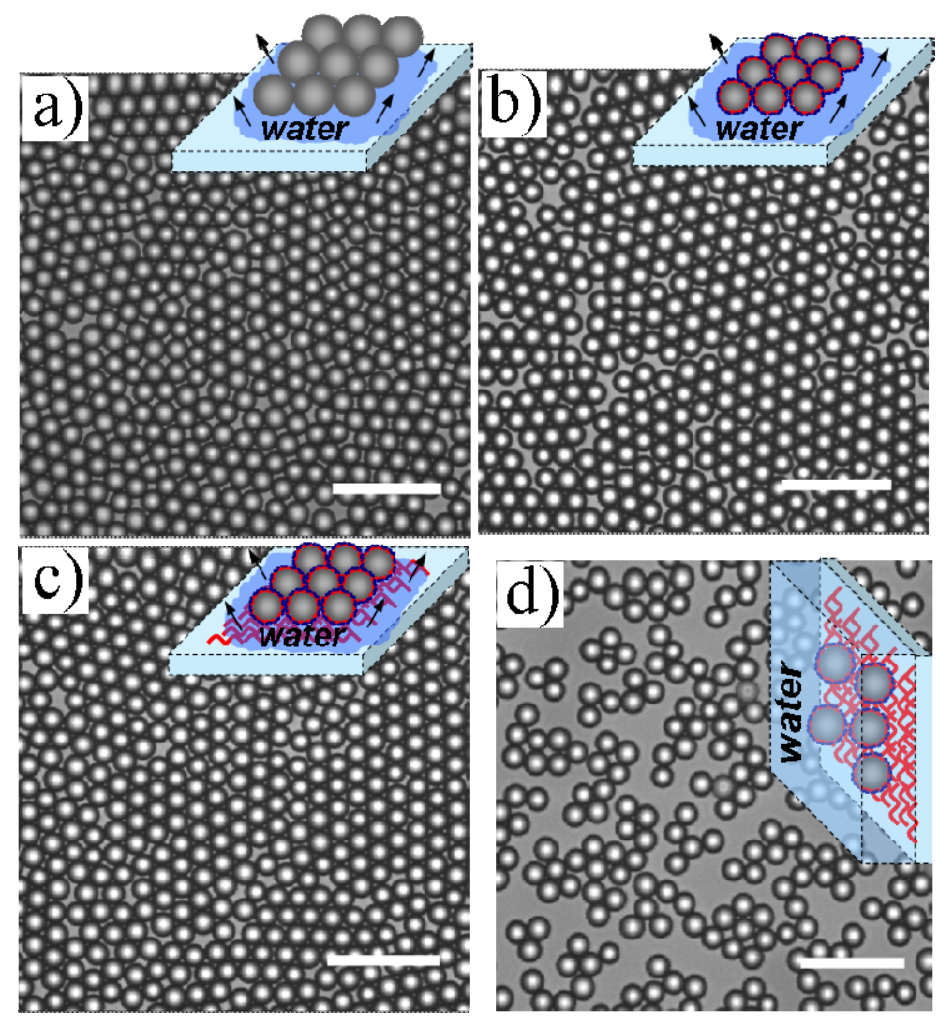

We further conducted adsorption of PEM coated silica spheres from a solution upon vertically withdrawing the substrate because it represents another method of adsorption and self-assembly. In fact, this approach is similar to Langmuir-Blodgett film deposition method. The particle distribution for such a case is shown in Figure 2(d). The particles form loose structures and large distances can be observed between them. Although it is possible to achieve higher density of coverage of particles in this case, the simplicity of evaporative self-assembly is certainly attractive. Therefore, adsorption of particles from solution significantly differs from that conducted using self-assembly on the substrate. The interaction between oppositely charged PEM colloids and substrate leads to adsorption of particles onto PEI and there exists no mechanism for self-assembly and close packing of these particles.

To further extend this work into 3D structures, we conducted self-assembly of a second layer of silica particles onto the first (self-assembled) layer of silica particles. The second layer of silica colloid coated PEM was fabricated by a similar procedure as that of the first layer. Briefly, a particle suspension was dropped on the top of the first sphere monolayer and left for drying. The particle distribution on the first PEM colloid layer can be seen in Figure 3(a). After evaporating and drying the 
aqueous suspension of the second layer, one can see that silica colloidal particles fill the voids between colloidal particles of the first layer which form a face centered cubic lattice.

Using polyelectrolytes possessing identical charges in both layers one can achieve intermixing of the top layer which actually flattens the upper surface. Such an intermixing is particularly interesting for colloidal particles with different sizes. Figure 3(b) presents such PEM coated particle self-assembly. Larger colloidal silica particles possessing PSS as the outer layer were adsorbed as described above. Subsequently, smaller coated colloids (also terminated with PSS) were allowed to become incorporated in the space between self-assembled larger colloidal particles (and were subsequently left for drying). As a result, small particles filled the inter-spaces between hexagonal close packed colloids of the first layer. During such an assembly, filling the space between larger particles by smaller particles can lead to smoothing and flattening of the colloidal particle layer surface. Such flattened substrates possessing 3D self-assembled structures are of interest for cell cultures, scaffolds and tissue engineering.

Figure 3. (a) Schematics (left) and top view in CLSM images (right) of a double layer self-assembly of PEM spheres. The right-hand side image shows an enlarged section of the middle panel. The scale bar corresponds to $20 \mu \mathrm{m}$. (b) Schematics (left) and top view in CLSM images (right) of self-assembly of a smaller polyelectrolyte coated silica colloidal particle array into the spaces between larger spheres. Red fluorescence originates from PAH-TRITC labeling of smaller silica particles. The scale bars correspond to $5 \mu \mathrm{m}$.

a)
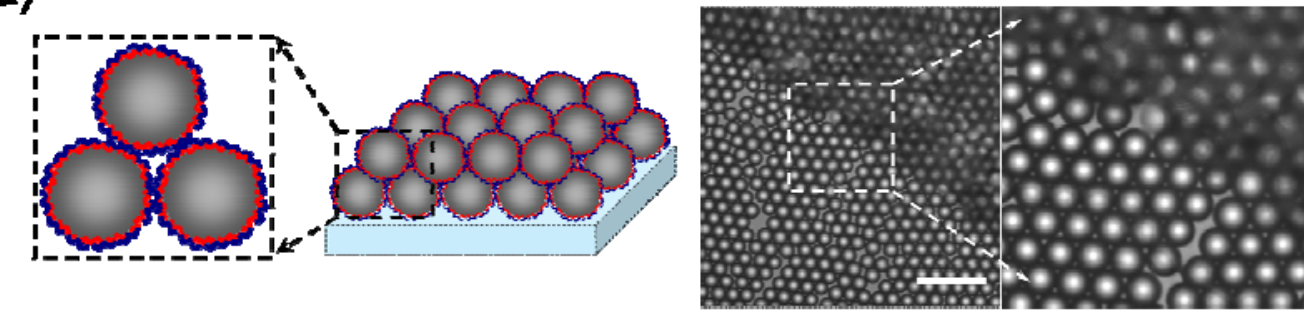

b)
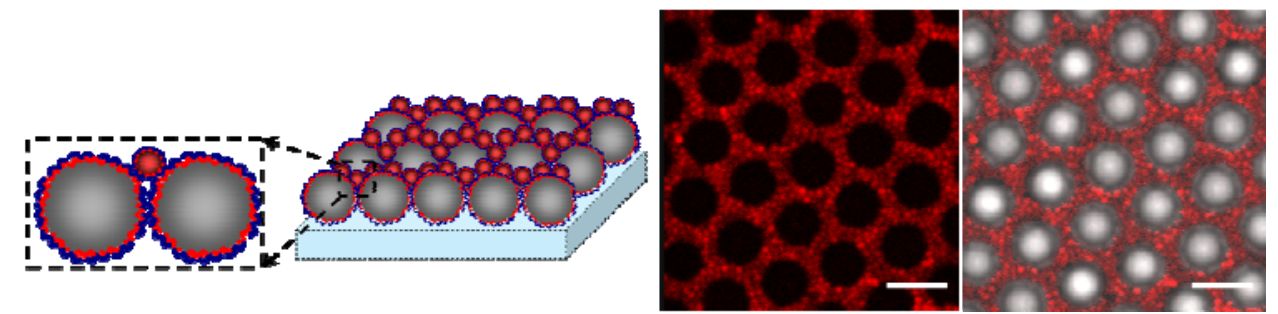

Modification of polyelectrolyte multilayers by nanoparticles and carbon nanotubes is of high interest due to their functionalization and potential applications. We perform these studies on flat surfaces by different methods: UV-vis for gold nanoparticles due to surface plasmon resonance effects; QCM in the case of magnetic nanoparticles because they lack characteristic UV-vis signatures and Raman spectroscopy for carbon nanotubes because they possess strong Raman signals. 


\subsection{Gold Nanoparticles on Polyelectrolyte Multilayers}

We further study assembly of gold nanoparticles onto planar surfaces of polyelectrolyte multilayer films. Nanoparticle/polyelectrolyte thin films may be exploited in diverse areas, including electrochemical sensing and colloidal crystals. Embedding gold nanoparticles into a polyelectrolyte shell results in change of the position of the optical stop band for colloid crystals fabricated from particles coated by a polyelectrolyte shell. Gold nanoparticles have an absorption peak in the visible part of the spectrum due to the surface plasmon resonance. The position of the peak depends on and can be controlled by the distance between nanoparticles or their aggregates. It can be noted that control of the distribution both at high [90] and low [91] concentrations of nanoparticles enables tailoring optical properties.

Measurements of absorption spectra of the obtained films showed that gold nanoparticles were successfully embedded onto polyelectrolyte multilayer films, as seen in Figure 4. Various types of gold nanoparticles were assembled onto the films: PNPs were produced at higher concentrations in comparison to NNPs. The latter have the surface plasmon resonance peak located at $525 \mathrm{~nm}$ (curve 1, Figure 4(a)). At such low concentration no aggregation is observed, although aggregation can be induced in this case by salt [41]. For positively charged nanoparticles the position of the maximum of the absorption band is located at $580 \mathrm{~nm}$ (curve 2 in Figure 4(a)). This indicates that some aggregation took place, most likely due to their high concentration. Even larger red-shift is observed for hc-PNPs, for which the peak is positioned at $615 \mathrm{~nm}$ (curve 3, Figure 4(a)). This shift is due to the stronger interaction of dipole moments on these, more aggregated, nanoparticles. This stronger interaction is attributed to decreasing the distances between neighboring nanoparticles as their concentration in the film increases. Results reported in this work extend our previous work on controlling the distribution of nanoparticles on spherical microcapsules [90,91] to planar LbL films. It can be noted that adsorption of one polyelectrolyte layer sequentially with nanoparticles [92] can be used for further increase of density of nanoparticles. AFM images of films containing PNPs and hc-PNPs gold nanoparticles are shown in Figure 4(b) and (c), respectively. Larger aggregates in the latter case can be clearly seen in these images.

Figure 4. (a) UV-vis absorption spectra of the polyelectrolyte films with one layer of: 1-NNPs; 2-PNPs; 3 -hc-PNPs; graphs are normalized to bring peaks for all curves within $30 \%$ of the unity. AFM images of polyelectrolyte films with two layers of positively charged gold nanoparticles: (b) PNPs; (c) hc-PNPs; the scan size in AFM images corresponds to $4 \times 4 \mu \mathrm{m}^{2}$.

a)

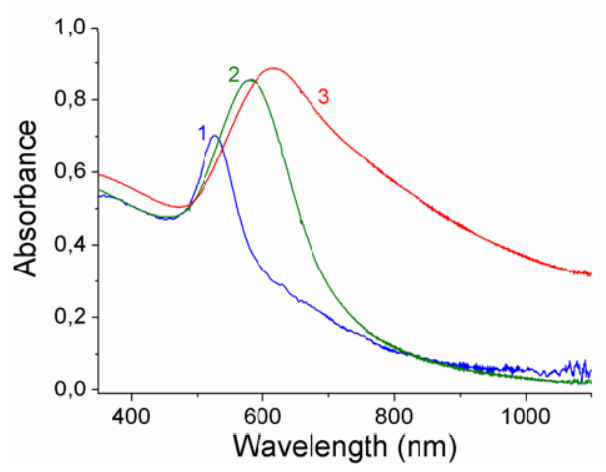

b)

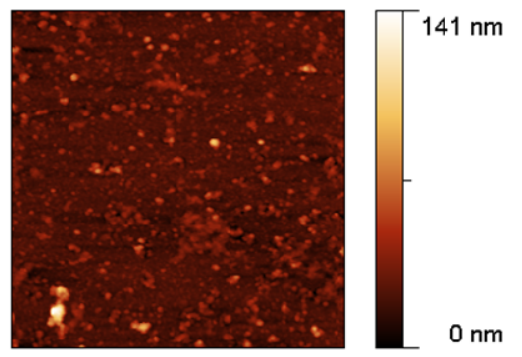

c)

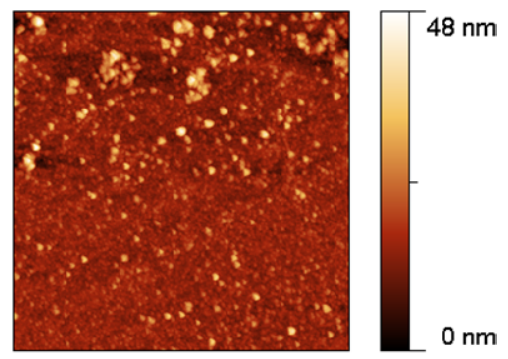




\subsection{Magnetic Nanoparticles on Polyelectrolyte Multilayers}

Magnetic nanoparticles represent another interesting component for manipulation of polyelectrolyte multilayers and therefore we used them for adsorption onto PEM films. UV-vis spectra will not provide the same information as for gold nanoparticles because of the absence of surface plasmon effects in oxide nanoparticles. Therefore, here we investigate the LbL assembly on planar substrates using the QCM technique.

The frequency change of the resonator with the number of polyelectrolyte and nanoparticle layers is presented in Figure 5. One layer of PEI was used as a precursor for all samples. It was found that PEI with high molecular weight improved the adsorption capabilities of the substrate for the LbL deposition. Data in Figure 5 present a typical frequency relationship for the LbL assembly. It can be concluded from these data that adsorption of positive magnetite nanoparticles was more effective in comparison to negative ones. Therefore, our results show that the iron oxide nanoparticles in the films form a sparse layer or can have heterogeneous distribution; hence the formation of some agglomerates is also possible.

Figure 5. (a) QCM measurement during deposition of polyelectrolyte layers and negative magnetic nanoparticles. (b) AFM and (c) TEM images of magnetite/polyelectrolyte film with negative nanoparticles, respectively. (d) QCM measurement during deposition of polyelectrolyte layers and positively charged magnetic nanoparticles. (e) AFM and (f) TEM images of magnetite/polyelectrolyte film with positively charged nanoparticles, respectively. The area of AFM scans is $5 \times 5 \mu \mathrm{m}^{2}$, the scale bars in the TEM images correspond to $50 \mathrm{~nm}$.

a)

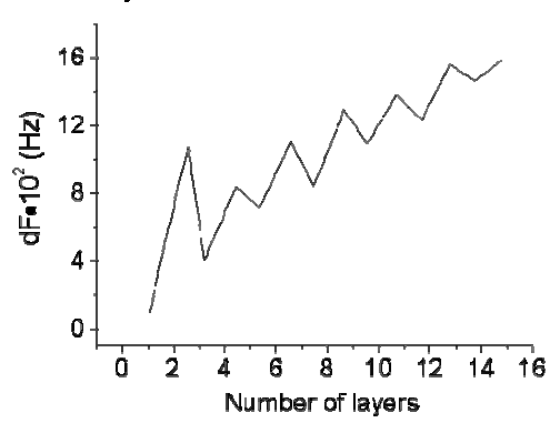

d)

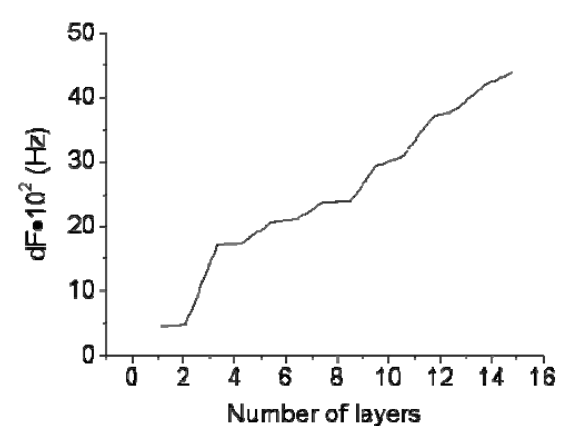

b)

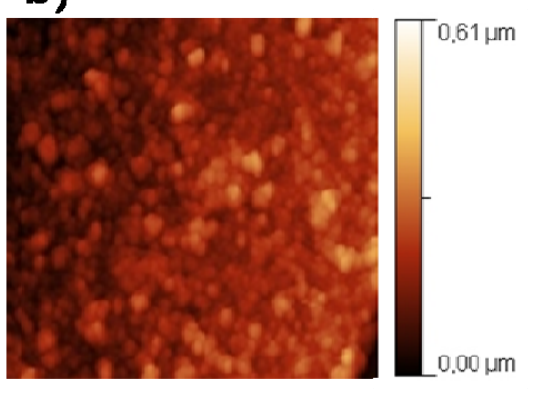

e)

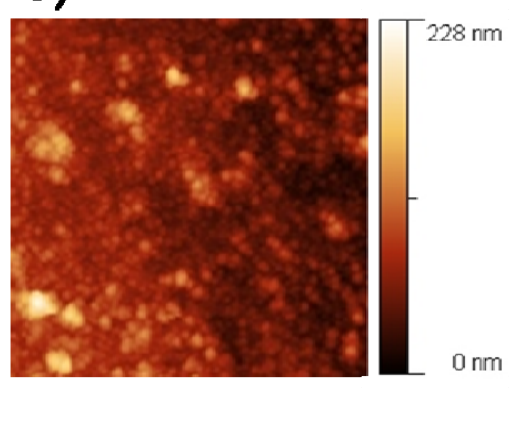

c)

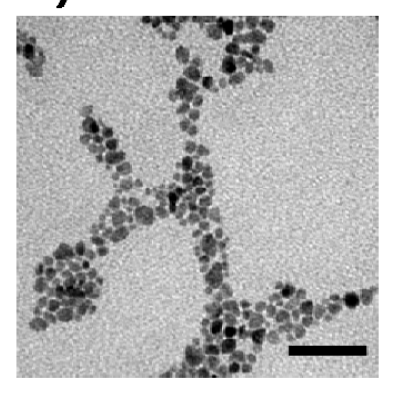

f)

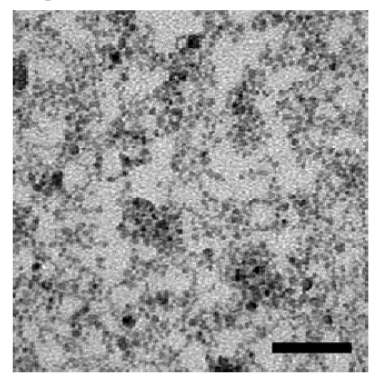


It can be concluded that adsorption of magnetic nanoparticles on polyelectrolyte multilayers takes place. In addition, it can be pointed out that the QCM technique represents an interesting method for monitoring adsorption of nanoparticles which lack a definite optical signature.

\subsection{Carbon Nanotubes on Polyelectrolyte Multilayers}

Carbon nanotubes represent an interesting component for inclusion into PEM because of their attractive opto-electronic, mechanical, optical and thermal properties. Originally hydrophobic, carbon nanotubes can not be directly dissolved in water and incorporated in LbL assembled layers. Therefore, a critical step of carbon nanotube adsorption is their dispersion in an aqueous solution. We used PSS polymers, which possess a hydrophobic benzene ring for dispersion of carbon nanotubes [93]; PSS is one of the most successful polymers used for solubilization of carbon nanotubes (capable of solubilizing carbon nanotubes at concentrations of up to $4 \mathrm{mg} / \mathrm{mL}$ ), however it can be salted out of the nanotubes at $\mathrm{NaCl}$ concentrations of over $15 \mathrm{mM}$ [93]. An alternative polymer for solubilization of carbon nanotubes is polyvinyl pirrolidone (PVP), which has even higher binding than PSS. PVP can also be salted out, but this requires concentrations of $\mathrm{NaCl}$ of over $115 \mathrm{mM}$. In both cases, salting out by divalent cation salts (for example, $\mathrm{MgCl}_{2}$ ) takes place at salt concentrations an order of magnitude lower.

Figure 6. Raman spectrum of films containing polyelectrolyte multilayers with multilayer MWCNTs; the inset shows schematics of the formation of such assembly.

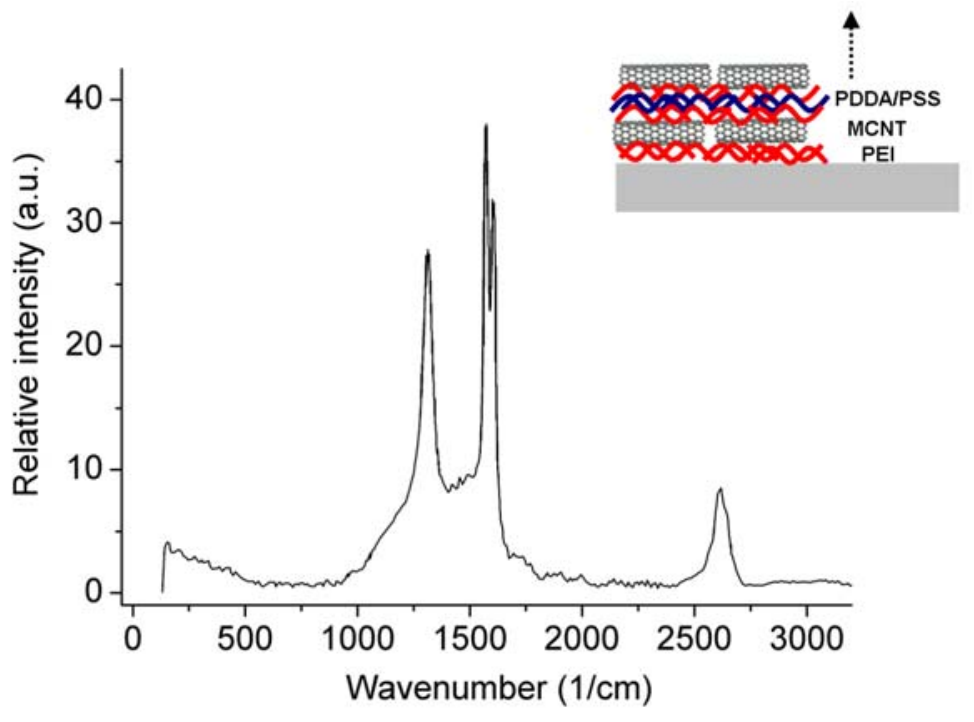

Although carbon nanotubes possess absorption in the visible-ultraviolet range of the electromagnetic spectrum, their broad absorption band lacks a well-defined peak in this spectral range. Therefore, we used Raman spectroscopy for characterization of carbon nanotube adsorption. Inclusion of carbon nanotubes into the polyelectrolyte films was confirmed by the Raman spectra of the films, Figure 6. Characteristic features of carbon nanotubes - strong peaks at around 1,350 and 1,580 cm which are attributed to the $\mathrm{D}$ and $\mathrm{G}$ bands, respectively — can be clearly seen in Figure 6. Also, other peaks at around 1,620 and 2,660 $\mathrm{cm}^{-1}$ can be observed, attributed to D' and G', respectively. A characteristic feature of MWCNTs, the peak at $1,620 \mathrm{~cm}^{-1}$, located next to the $\mathrm{G}$ band $\left(\right.$ at $\left.1,580 \mathrm{~cm}^{-1}\right)$, is clearly seen in Figure 6. Adsorption of MWCNTs on polyelectrolyte multilayer can be unambiguously 
concluded from these data. We note that broad absorption of carbon nanotubes in the visible spectral range [61-64] and their high light-to-heat conversion efficiency [67] makes them an attractive alternative to non-spherical nanoparticles [94] and nanorods [95,96] in regard to controlling the permeability of polymeric microcapsules [37-43] and activation of planar films [21-23]. We note that results of our work can be used for producing polyelectrolyte multilayers simultaneously functionalized not only with gold and magnetic nanoparticles [97], but also with these nanoparticles and carbon nanotubes. Furthermore, these results can be used for self-assembling particle-based structures as well as for building spherical microcapsules and planar films with a variety of polymers [98] and for increasing multifunctionality of more sophisticated hierarchies of microcapsules [99].

\section{Conclusions}

Polyelectrolytes are presented for building spherical particles and planar films functionalized with gold and magnetic nanoparticles as well as carbon nanotubes. Evaporative self-assembly of silica particles is studied and effects of polyelectrolytes on this process are reported: polyelectrolyte coatings are found to produce a minor effect upon self-assembly of silica particles onto a glass substrate. Evaporation of solvent is found to produce self-assembled particles as opposed to adsorption of polyelectrolyte coated silica particles onto a vertically situated substrate in an aqueous solution wherein self-assembly is not achieved. Double and multiple layer formation of silica particles demonstrated in this work indicate that some practical structures functionalized with a variety of materials can be built using this approach. It is shown that smaller colloidal particles fill the gaps in the self-assembled arrays of larger colloidal silica particles, thus demonstrating that the upper surface of such arrays can be flattened for further build-up of assemblies. The concentration of gold nanoparticles within polyelectrolyte multilayer films was controlled, while magnetic nanoparticles as well as carbon nanotubes are also adsorbed. The results of this work show that polyelectrolytes as well as polyelectrolytes together with nanoparticles and nanotubes can be used for building sophisticated spherical and planar structures.

\section{Acknowledgements}

We thank Anneliese Heilig for the AFM measurements. I. Marchenko thanks a FEBS scholarship for support; O. Inozemtseva thanks the DAAD for support.

\section{References}

1. Skirtach, A.G.; Kreft, O. Stimuli-sensitive nanotechnology for drug delivery. In Biotechnology: Pharmaceutical Aspects; de Velliers, M.M., Aramwit, P., Kwon, G.S., Eds.; Springer: New York, NY, USA, 2009; Volume 10, pp. 545-578.

2. Decher, G. Fuzzy nanoassemblies: Toward layered polymeric multicomposites. Science 1997, 277, 1232-1237.

3. Lvov, Y.; Decher, G.; Möhwald, H. Assembly, structural characterization, and thermal-behavior of layer-by-layer deposited ultrathin films of poly(vinyl sulfate) and poly(ally amine). Langmuir 1993, 9, 481-486. 
4. Caruso, F.; Furlong, D.N.; Ariga, K.; Ichinose, I.; Kunitake, T. Characterization of polyelectrolyte-protein multilayer films by atomic force microscopy, scanning electron microscopy, and Fourier transform infrared reflection-absorption spectroscopy. Langmuir 1998, 14, 4559-4565.

5. Sukhorukov, G.B.; Donath, E.; Davis, S.; Lichtenfeld, H.; Caruso, F.; Popov, V.I.; Mohwald, H. Stepwise polyelectrolyte assembly on particle surfaces: A novel approach to colloid design. Polym. Adv. Technol. 1998, 9, 759-767.

6. Kharlampieva, E.; Slocik, J.M.; Tsukruk, T.; Naik, R.R.; Tsukruk, V.V. Polyaminoacid-induced growth of metal nanoparticles on layer-by-layer templates. Chem. Mater. 2008, 20, 5822-5831.

7. Wandrey, C.; Hunkeler, D.; Wendler, U.; Jaeger, W. Counterion activity of highly charged strong polyelectrolytes. Macromolecules 2000, 33, 7136-7143.

8. Malinova, V.; Wandrey, C. Loading polyelectrolytes onto porous microspheres: Impact of molecular and electrochemical parameters. J. Phys. Chem. B 2007, 111, 8494-9501.

9. Jessel, N.; Alatar, F.; Lavalle, P.; Mutterer, J.; Decher, G.; Shaaf, P.; Voegel, J.C.; Odier, J. Bioactive coatings based on a polyelectrolyte multilayer architecture functionalized by embedded proteins. Adv. Mater. 2003, 15, 692-695.

10. Benkriane-Jessel, N.; Schwinte, P.; Falvey, P.; Darcy, R.; Haikel, Y.; Shaaf, P.; Voegel, J.C.; Odier, J. Build-up of polypeptide multilayer coatings with anti-inflammatory properties based on the embedding of piroxicam-cyclodextrin complexes. Adv. Funct. Mater. 2004, 14, 174-182.

11. Jessel, N.B.; Schwinte, P.; Donohue, R.; Lavalle, P.; Boulmedias, F.; Szalontai, P.; Darcy, R.; Voegel, J.C.; Odier, J. Pyridylamino-beta-cyclodextrin as a molecular chaperone for lipopolysaccharide embedded in a multilayered polyelectrolyte architecture. Adv. Funct. Mater. 2004, 14, 963-969.

12. Benkriane-Jessel, N.; Lavalle, P.; Hurbsh, E.; Holl, V.; Senger, B.; Haikel, Y.; Voegel, J.C.; Odier, J.; Shaaf, P. Short-time timing of the biological activity of functionalized polyelectrolyte multilayers. Adv. Funct. Mater. 2005, 15, 648-654.

13. Zhang, X.; Sharma, K.K.; Boeglin, M.; Ogier, J.; Mainard, D.; Voegel, J.C.; Mely, Y.; Benkirane-Jessel, N. Transfection ability and intracellular DNA pathway of nanostructured gene-delivery systems. Nano Lett. 2008, 13, 2432-2436.

14. Benkriane-Jessel, N.; Lavalle, P.; Meyer, F.; Audouin, F.; Frisch, B.; Shaaf, P.; Decher, G.; Voegel, J.C.; Odier, J. Control of monocyte morphology on and response to model surfaces for implants equipped with anti-inflammatory agents. Adv. Mater. 2004, 16, 1507-1511.

15. Dierich, A.; Guen, E.L.; Messaddeq, N.; Stoltz, J.F.; Netter, P.; Shaaf, P.; Voegel, J.C.; Benkriane-Jessel, N. Bone formation mediated by synergy-acting growth factors embedded in a polyelectrolyte multilayer film. Adv. Mater. 2007, 19, 693-697.

16. Facca, S.; Cortez, C.; Mendoza-Palomares, C.; Messadeq, N.; Dierich, A.; Johnston, A.P.R.; Mainard, D.; Voegel, J.-C.; Caruso, F.; Benkirane-Jessel, N. Active multilayered capsules for in vivo bone formation. Proc. Nat. Acad. Sci. 2010, 107, 3406-3411.

17. Fioretti, F.; Mendoza-Palomares, C.; Helms, M.; Alam, D.A.; Richert, L.; Arntz, Y.; Rinckenbach, S.; Garnier, F.; Ha1kel, Y.; Gangloff, S.C.; Benkirane-Jessel, N. Nanostructured assemblies for dental application. ACS Nano 2010, 4, 3277-3287. 
18. Picart, C.; Lavalle, P.; Hubert, P.; Cuisinier, F.J.G.; Decher, G.; Schaaf, P.; Voegel, J.C. Buildup mechanism for poly(L-lysine)/hyaluronic acid films onto a solid surface. Langmuir 2001, 17, 7414-7424.

19. Mjahed, H.; Porcel, C.; Senger, B.; Chassepot, A.; Netter, P.; Gillet, P.; Decher, G.; Voegel, J.C.; Schaaf, P.; Benkirane-Jessel, N.; Boulmedias, F. Micro-stratified architectures based on successive stacking of alginate gel layers and poly(L-lysine)-hyaluronic acid multilayer films aimed at tissue engineering. Soft Matter 2008, 4, 1422-1429.

20. Boulmedais, F.; Ball, V.; Schwinte, P.; Frisch, B.; Schaaf, P.; Voegel, J.C. Buildup of exponentially growing multilayer polypeptide films with internal secondary structure. Langmuir 2003, 19, 440-445.

21. Volodkin, D.V.; Delcea, M.; Mohwald, H.; Skirtach, A.G. Remote near-IR light activation of a hyaluronic acid/poly(l-lysine) multilayered film and film-entrapped microcapsules. ACS Appl. Mater. Interfaces 2009, 1, 1705-1710.

22. Skirtach, A.G.; Volodkin, D.V.; Mohwald, H. Bio-interfaces-Interaction of PLL/HA thick films with nanoparticles and microcapsules. Chem. Phys. Chem. 2010, 11, 822-829.

23. Volodkin, D.V.; Madaboosi, N.; Blacklock, J.; Skirtach, A.G.; Moehwald, H. Surface-supported multilayers decorated with bio-active material aimed at light-triggered drug delivery. Langmuir 2009, 25, 14037-14043.

24. De Geest, B.G.; De Kokker, S.; Sukhorukov, G.B.; Kreft, O.; Parak, W.J.; Skirtach, A.G.; Demeester, J.; De Smedt, S.C.; Hennink, W.E. Polyelectrolyte microcapsules for biomedical applications. Soft Matter 2009, 5, 282-291.

25. Becker, A.L.; Johnston, A.P.R.; Caruso, F. Layer-by-layer-assembled capsules and films for therapeutic delivery. Small 2010, 6, 1836-1852.

26. De Cock, L.J.; De Koker, S.; De Geest, B.G.; Grooten, J.; Vervaet, C.; Remon, J.P.; Sukhorukov, G.B.; Antipina, M.N. Polymeric multilayer capsules in drug delivery. Angew. Chem. Int. Ed. 2010, 49, 6954-6973.

27. Rivera Gil, P.; Parak, W.J. Composite nanoparticles take aim at cancer. ACS Nano 2008, 2, 2200-2205.

28. Rivera Gil, P.; del Mercato, L.L.; del-Pino, P.; Munoz-Javier, A.; Parak, W.J. Nanoparticle-modified polyelectrolyte capsules. Nano Today 2008, 3, 12-21.

29. Matsuaki, M.; Akashi, M. Functional multilayered capsules for targeting and local drug delivery. Expert Opin. Drug Deliv. 2009, 6, 1207-1217.

30. Delcea, M.; Mohwald, H.; Skirtach, A.G. Stimuli responsive LbL capsules and nanoshells for drug delivery. Adv. Drug Deliv. Rev. 2011, 63, in press.

31. Orozco, V.H.; Kozlovskaya, V.; Kharlampieva, E.; López, B.L.; Tsukruk, V.V. Biodegradable self-reporting nanocomposite films of poly(lactic acid) nanoparticles engineered by layer-by-layer assembly. Polymer 2010, 51, 4127-4139.

32. Grohn, F. Soft matter nanoparticles with various shapes and functionalities can form through electrostatic self-assembly. Soft Matter 2010, 6, 4296-4302.

33. Kozlovskaya, V.; Kharlampieva, E.; Khanal, B.P.; Manna, P.; Zubarev, E.R.; Tsukruk, V.V. Ultrathin layer-by-layer hydrogels with incorporated gold nanorods as $\mathrm{pH}$-sensitive optical materials. Chem. Mater. 2008, 20, 7474-7485. 
34. Tokarev, I.; Tokareva, I.; Gopishetty, V.; Katz, E.; Minko S. Specific biochemical-to-optical signal transduction by responsive thin hydrogel films loaded with noble metal nanoparticles. Adv. Mater. 2010, 22, 1412-1416.

35. Bedard, M.F.; Munoz-Javier, A.; Mueller, R.; del Pino, P.; Fery, A.; Parak, W.J.; Skirtach, A.G.; Sukhorukov, G.B. On the mechanical stability of polymeric microcontainers functionalized with nanoparticles. Soft Matter 2009, 5, 148-155.

36. Delcea, M.; Schmidt, S.; Palankar, R.; Fernandes, P.A.L.; Fery, A.; Mohwald, H.; Skirtach, A.G. Mechanobiology: Correlation between mechanical stability of microcapsules studied by AFM and impact of cell-induced stresses. Small 2010, doi: 10.1002/smll.201001478.

37. Skirtach, A.G.; Antipov, A.A.; Shchukin, D.G.; Sukhorukov, G.B. Remote activation of capsules containing Ag nanoparticles and IR dye by laser light. Langmuir 2004, 20, 6988-6992.

38. Radt, B.; Smith, T.A.; Caruso, F. Optically addressable nanostructured capsules. Adv. Mater. 2004, 16, 2184-2187.

39. Skirtach, A.G.; Dejugnat, C.; Braun, D.; Susha, A.S.; Rogach, A.L.; Parak, W.J.; Mohwald, H.; Sukhorukov, G.B. The role of metal nanoparticles in remote release of encapsulated materials. Nano Lett. 2005, 5, 1371-1377.

40. Angelatos, A.S.; Radt, B.; Caruso, F. Light-responsive polyelectrolyte/gold nanoparticle microcapsules. J. Phys. Chem. B 2005, 109, 3071-3076.

41. Bedard, M.F.; Braun, D.; Sukhorukov, G.B.; Skirtach, A.G. Toward self-assembly of nanoparticles on polymeric microshells: Near-IR release and permeability. ACS Nano 2008, 2, 1807-1816.

42. Bedard, M.F.; De Geest, B.G.; Moehwald, H.; Sukhorukov, G.B.; Skirach, A.G. Direction specific release from giant microgel-templated polyelectrolyte microcontainers. Soft Matter 2009, 5, 3927-3931.

43. Skirtach, A.G.; Javier AM, Kreft, O.; Kohler, K.; Alberola, A.P.; Mohwald, H.; Parak, W.J.; Sukhorukov, G.B. Laser-induced release of encapsulated materials inside living cells. Angew. Chem. Int. Ed. 2006, 45, 4612-4617.

44. Palankar, R.; Skirtach, A.G.; Kreft, O.; Bédard, M.; Garstka, M.; Gould, K.; Möhwald, H.; Sukhorukov, G.B.; Winterhalter, M.; Springer, S. Controlled intracellular release of peptides from microcapsules enhances antigen presentation on MHC class I molecules. Small 2009, 5, 2168-2176.

45. Lee, S.E.; Sasaki, D.Y.; Perroud, T.D.; Yoo, D.; Patel, K.D.; Lee, L.P. Biologically functional cationic phospholipid-gold nanoplasmonic carriers of RNA. J. Am. Chem. Soc. 2009, 131, 14066-14074.

46. Alessandri, I. Plasmonic heating assisted deposition of bare $\mathrm{Au}$ nanoparticles on titania nanoshells. J. Colloid Interface Sci. 2010, 351, 576-579.

47. Alessandri, I. Writing, self-healing, and self-erasing on conductive pressure-sensitive adhesives. Small 2010, 6, 1679-1685.

48. Yu, J.; Javier, D.; Yaseen, M.A.; Nitin, N.; Richards-Kortum, R.; Anvari, B.; Wong, M.S. Self-assembly synthesis, tumor cell targeting, and photothermal capabilities of antibody-coated indocyanine green nanocapsules. J. Am. Chem. Soc. 2010, 132, 1929-1938. 
49. Hrelescu, C.; Stehr, J.; Ringler, M.; Sperling, R.A.; Parak, W.J.; Klar, T.A.; Feldman, J. DNA melting in gold nanostove clusters. J. Phys. Chem. C 2010, 114, 7401-7411.

50. Baffou, G.; Quidant, R.; de Abajo, F.J.G. Nanoscale control of optical heating in complex plasmonic systems. ACS Nano 2010, 4, 709-716.

51. Terentyuk, G.S.; Maslyakova, G.N.; Suleymanova, L.V.; Khlebtsov, N.G.; Khlebtsov, B.N.; Akchurin, G.G.; Maksimova, I.L.; Tuchin, V.V. Laser-induced tissue hyperthermia mediated by gold nanoparticles: Toward cancer phototherapy. J. Biomed. Opt. 2009, 14, 021016.

52. Anderson, L.J.E.; Hansen, E.; Lukianova-Hleb, E.Y.; Hafner, J.H.; Lapotko, D.O. Optically guided controlled release from liposomes with tunable plasmonic nanobubbles. J. Control. Release 2010, 144, 151-158.

53. You, J.; Shao, R.P.; Wei, X.; Gupta, S.; Li, C. Near-infrared light triggers release of paclitaxel from biodegradable microspheres: photothermal effect and enhanced antitumor activity. Small 2010, 6, 1022-1031.

54. Agasti, S.S.; Chompoosor, A.; You, C.C.; Ghosh, P.; Kim, C.K.; Rotello, V.M. Photoregulated release of caged anticancer drugs from gold nanoparticles. J. Am. Chem. Soc. 2009, 131, 5728-5729.

55. Yan, W.Y.; Li, C.M. Direct modulation of localized surface plasmon coupling of $\mathrm{Au}$ nanoparticles on solid substrates via weak polyelectrolyte-mediated layer-by-layer self assembly. Langmuir 2009, 25, 7578-7585.

56. Russell, A.G.; McKnight, M.D.; Sharp, A.C.; Hestekin, J.A.; Roper, D.K. Gold nanoparticles allow optoplasmonic evaporation from open silica cells with a logarithmic approach to steady-state thermal profiles. J. Phys. Chem. C 2010, 114, 10132-10139.

57. Barman, A.K.; Verma, S. Sunlight mediated disruption of peptide-based soft structures decorated with gold nanoparticles. Chem. Commun. 2010, 46, 6992-6994.

58. Gorin, D.A.; Portnov, S.A.; Inozemtseva, O.A.; Luklinska, Z.; Yashchenok, A.M.; Pavlov, A.M.; Skirach, A.G.; Mohwald, H.; Sukhorukov, G.B. Magnetic/gold nanoparticle functionalized biocompatible microcapsules with sensitivity to laser irradiation. Phys. Chem. Chem. Phys. 2008, 10, 6899-6905.

59. Katagiri, K.; Nakamura, M.; Koumoto, K. Magnetoresponsive smart capsules formed with polyelectrolytes, lipid bilayers and magnetic nanoparticles. ACS Appl. Mater. Interfaces 2010, 2, 768-773.

60. Jiang, C.; Ko, H.; Tsukruk, V.V. Strain-sensitive Raman modes of carbon nanotubes in deflecting freely suspended nanomembranes. Adv. Mater. 2005, 17, 2127-2131.

61. Ko, H.; Jiang, C.; Shulha, H.; Tsukruk, V.V. Carbon nanotube arrays encapsulated into freely suspended flexible films. Chem. Mater. 2005, 17, 2490-2493.

62. Patine, S.J.; Okawa, D.; Zettl, A.; Frechet. J.M.J. Chemicals on demand with phototriggerable microcapsules. J. Am. Chem. Soc. 2009, 131, 13586-13587.

63. Choi, W.S.; Yang, H.M.; Koo, H.Y.; Lee, H.J.; Lee, Y.B.; Bae, T.S.; Jeon, I.C. Smart microcapsules encapsulating reconfigurable carbon nanotube cores. Adv. Funct. Mater. 2010, 20, 820-825.

64. Yashchenok, A.M.; Bratashov, D.N.; Gorin, D.A.; Lomova, M.V.; Pavlov, A.M.; Sapelkin, A.V.; Shim, B.S.; Khomutov, G.B.; Kotov, N.A.; Sukhorukov, G.B.; Möhwald, H.; Skirtach, A.G. 
Carbon nanotubes on polymeric microcapsules: Free-standing structures and point-wise laser openings. Adv. Funct. Mater. 2010, 20, 3136-3142.

65. Paunov, V.N.; Panhuis, M.I.H. Fabrication of carbon nanotube-based microcapsules by a colloid templating technique. Nano Technol. 2005, 16, 1522-1525.

66. Ji, Q.M.; Yoon, S.B.; Hill, J.P.; Vinu, A.; Yu, J.S.; Ariga, K.S. Layer-by-layer films of dual-pore carbon capsules with designable selectivity of gas adsorption. J. Am. Chem. Soc. 2009, 131, 4220-4221.

67. Shen, Y.F.; Skirtach, A.G.; Seki, T.; Yagai, S.; Li, H.G.; Mohwald, H.; Nakanishi, T. Assembly of fullerene-carbon nanotubes: temperature indicator for photothermal conversion. J. Am. Chem. Soc. 2010, 132, 8566-8568.

68. Nie, Z.H.; Petukhova, A.; Kumacheva, E. Properties and emerging applications of self-assembled structures made from inorganic nanoparticles. Nat. Nanotechnol. 2010, 5, 15-25.

69. Wang, D.; Caruso, F. Fabrication of polyaniline inverse opals via templating ordered colloidal assemblies. Adv. Mater. 2001, 13, 350-35.

70. Lotsch, B.V.; Knobbe, C.B.; Ozin, G.A. A step towards optically encoded silver release in 1D photonic crystals. Small 2009, 5, 1498-1503.

71. Arsenault, A.C.; Puzzo, D.P.; Manners, I.; Ozin, G.A. Photonic-crystal full-colour displays. Nat. Photonic. 2007, 1, 468-472.

72. Min, W.L.; Jiang, P.; Jiang, B. Large-scale assembly of colloidal nanoparticles and fabrication of periodic subwavelength structures. Nano Technol. 2008, 19, 475604.

73. Jiang, P.; McFarland, M.J. Large-scale fabrication of wafer-size colloidal crystals, macroporous polymers and nanocomposites by spin-coating. J. Am. Chem. Soc. 2004, 126, 13778-13786.

74. Braun, P.V.; Wiltzius, P. Microporous materials-Electrochemically grown photonic crystals. Nature 1999, 402, 603-604.

75. Xie, Z.Y.; Sun, L.G.; Han, G.Z.; Gu, Z.Z. Optical switching of a birefringent photonic crystal. Adv. Mater. 2008, 20, 3601-3604.

76. Bardosova, M.; Pemble, M.E.; Povey, I.M.; Tredgold, R.H. The Langmuir-Blodgett approach to making colloidal photonic crystals from silica spheres. Adv. Mater. 2010, 22, 3104-3124.

77. Hong, S.W.; Byun, M.; Lin, Z. Robust self-assembly of highly ordered complex structures by controlled evaporation of confined microfluids. Angew. Chem. Int. Ed. 2009, 48, 512-516.

78. Briseno, A.L.; Han, S.; Rauda, I.E.; Zhou, F.M.; Toh, C.S.; Nemanick, E.J.; Lewis, N.S. Electrochemical polymerization of aniline monomers infiltrated into well-ordered truncated eggshell structures of polyelectrolyte multilayers. Langmuir 2004, 20, 219-226.

79. Bhattacharya, A.; Balazs, A.C. Designing microcapsule arrays that propagate chemical signals. Phys. Rev. E 2010, 82, 021801.

80. Park, J.Y.; Ponnapati, R.; Taranekar, P. Advincula, R.C. Carbazole peripheral poly(benzyl ether) dendrimers at the air-water interface: Electrochemical cross-linking and electronanopatterning. Langmuir 2010, 26, 6167-6176.

81. Fournier, D.; De Geest, B.G.; Du Prez, F.E. On-demand click functionalization of polyurethane films and foams. Polymer 2009, 50, 5362-5367. 
82. Alves, N.M.; Picart, C.; Mano, J.F. Self assembling and crosslinking of polyelectrolyte multilayer films of chitosan and alginate studied by QCM and IR spectroscopy. Macromol. BioSci. 2009, 9, 776-785.

83. Tokarev, I.; Minko, S. Stimuli-responsive porous hydrogels at interfaces for molecular filtration, separation, controlled release, and gating in capsules and membranes. Adv. Mater. 2010, 22, 3446-3462.

84. Staedler, B.; Chandrawati, R.; Price, A.D.; Chong, S.F.; Breheney, S.F.; Postma, A.; Connal, L.A.; Zelikin, A.N.; Caruso, F. A microreactor with thousands of subcompartments: enzyme-loaded liposomes within polymer capsules. Angew. Chem. Int. Ed. 2009, 48, 4359-4362.

85. Yashchenok, A.M.; Delcea, M.; Videnova, K.; Jares-Erijman, E.A.; Jovin, T.M.; Konrad, M.; Mohwald, H.; Skirtach, A.G. Enzyme reaction in the pores of $\mathrm{CaCO}_{3}$ particles upon ultrasound disruption of attached substrate-filled liposomes. Angew. Chem. Int. Ed. 2010, 49, 8116-8120.

86. Petrov, A.I.; Volodkin, D.V.; Sukhorukov, G.B. Protein-calcium carbonate coprecipitation: A tool for protein encapsulation. Biotechnol. Prog. 2005, 21, 918-925.

87. Gittins, D.; Caruso, F. Spontaneous phase transfer of nanoparticulate metals from organic to aqueous media. Angew Chem. Int. Ed. 2001, 40, 3001-3004.

88. Massart, R. Preparation of aqueous magnetic liquids in alkaline and acidic media. IEEE Trans. Magn. 1981, 17, 1247-1248.

89. Reinhardt, K.A.; Kern, W. Handbook of Semiconductor Wafer Cleaning Technology; William Andrew Inc.: New York, NY, USA, 2007.

90. Skirtach, A.G.; Dejugnat, C.; Braun, D.; Susha, A.S.; Rogach, A.L.; Sukhorukov, G.B. Nanoparticles distribution control by polymers: Aggregates versus nonaggregates. J. Phys. Chem. C 2007, 111, 555-564.

91. Parakhonskiy, B.; Bedard, M.F.; Bukreeva, T.V.; Sukhorukov, G.B.; Mohwald, H.; Skirtach, A.G. Nanoparticles on polyelectrolytes at low concentration: controlling concentration and size. $J$. Phys. Chem. C 2010, 114, 1996-2002.

92. De Geest, B.G.; Skirtach, A.G.; De Beer, T.R.M.; Sukhorukov, G.B.; Bracke, L.; Baeyens, W.R.G.; Demeester, J.; De Smedt, S.C. Stimuli-responsive multilayered hybrid nanoparticle/polyelectrolyte capsules. Macromol. Rapid Commun. 2007, 28, 88-95.

93. O’Connell, M.J.; Boul, P.; Ericson, L.M.; Huffman, C.; Wang, Y.H.; Haroz, E.; Kuper, C.; Tour, J.; Ausman, K.D.; Smalley, R.E. Reversible water-solubilization of single-walled carbon nanotubes by polymer wrapping. Chem. Phys. Lett. 2001, 342, 265-271.

94. Sau, T.K.; Rogach, A.L.; Jackel, F.; Klar, T.A.; Feldmann, J. Properties and applications of colloidal nonspherical noble metal nanoparticles. Adv. Mater. 2010, 22, 1805-1825.

95. Guo, R.; Zhang, L.Y.; Qian, H.Q.; Li, R.T.; Jiang, X.Q.; Liu, B.R. Multifunctional nanocarriers for cell imaging, drug delivery, and near-ir photothermal therapy. Langmuir 2010, 26, 5428-5434.

96. Huang, H.C.; Rege, K.; Heys, J.J. Spatiotemporal temperature distribution and cancer cell death in response to extracellular hyperthermia induced by gold nanorods. ACS Nano 2010, 4, 2892-2900.

97 Gorin, D.A.; Portnov, S.A.; Inozemtseva, O.A.; Luklinska, Z.; Yashchenok, A.M.; Pavlov, A.M.; Skirach, A.G.; Mohwald, H.; Sukhorukov, G.B. Magnetic/gold nanoparticle functionalized 
biocompatible microcapsules with sensitivity to laser irradiation. Phys. Chem. Chem. Phys. 2008, 10, 6899-6905.

98. Szarpak, A.; Cui, D.; Dubreuil, F.; De Geest, B.G.; De Cock, L.J.; Picart, C.; Auzely-Velty, R. Designing hyaluronic acid-based layer-by-layer capsules as a carrier for intracellular drug delivery. Biomacromolecules 2010, 11, 713-720.

99. Delcea, M.; Yashchenok, A.; Videnova, K.; Kreft, O.; Mohwald, H.; Skirtach, A.G. Multicompartmental micro- and nanocapsules: Hierarchy and applications in biosciences. Micromol. Biosci. 2010, 10, 465-474.

(C) 2010 by the authors; licensee MDPI, Basel, Switzerland. This article is an open access article distributed under the terms and conditions of the Creative Commons Attribution license (http://creativecommons.org/licenses/by/3.0/). 